\title{
SUSY Dark Matter In Light Of CDMS/XENON Limits
}

\author{
Jin Min Yang \\ Key Laboratory of Frontiers in Theoretical Physics, \\ Institute of Theoretical Physics, Academia Sinica, Beijing 100190, China
}

\begin{abstract}
In this talk we briefly review the current CDMS/XENON constraints on the neutralino dark matter in three popular supersymmetric models: the minimal (MSSM), the next-to-minimal (NMSSM) and the nearly minimal (nMSSM). The constraints from the dark matter relic density and various collider experiments are also taken into account. The conclusion is that for each model the current CDMS/XENON limits can readily exclude a large part of the parameter space allowed by other constraints and the future SuperCDMS or XENON100 can cover most of the allowed parameter space. The implication for the Higgs search at the LHC is also discussed. It is found that in the currently allowed parameter space the MSSM charged Higgs boson is quite unlikely to be discovered at the LHC while the neutral Higgs bosons $H$ and $A$ may be accessible at the LHC in the parameter space with a large $\mu$ parameter.
\end{abstract}




\section{INTRODUCTION}

Low energy supersymmetry (SUSY) is the mainstream of new physics beyond the standard model and often called the standard theory for non-standard (new) physics. In addition to solve the fine-tuning problem and ensure the unification of the gauge couplings, the SUSY models (with $R$-parity) also have a pleasant byproduct- the cosmic dark matter. The lightest supersymmetric particle, which is usually assumed to be the lightest neutralino (albeit gravitino may equally be qualified), is a natural candidate for the cosmic cold dark matter. Such a neutralino serves as a perfect WIMP (Weakly Interacting Massive Particle) and is very likely within the sensitivity reach of the dark matter direct detection experiments like the CDMS [1] or XENON [2].

The current results from CDMS and XENON have set some limits on the strength of the dark matter-nucleon scattering [1, 2]. These limits can be transferred to the constraints on the SUSY parameter space under the assumption that the cosmic dark matter is composed of the neutralino. Recently, in our studies [3, 4] (for earlier studies, see, e.g., [5]) a scan over the SUSY parameter space was performed by considering the new CDMS/XENON limits together with the constraints from the dark matter relic density and various collider experiments like the search for the Higgs boson and super particles, the precision electroweak measurements and the muon anomalous magnetic moment. For comparison, several SUSY models were taken in these studies, which are the minimal (MSSM), the next-to-minimal (NMSSM) ) [6] and the nearly minimal (nMSSM) [7].

In the meantime, the Large Hadron Collider at CERN will intensively hunt for the SUSY particles including the neutralino dark matter and the SUSY Higgs bosons (SUSY predicts at least five Higgs bosons, i.e., in addition to the SM-like Higgs boson $h_{S M}$, there are the neutral $H, A$ and the charged $H^{ \pm}$). Given a framework of SUSY model, the dark matter search experiments like CDMS or XENON and the Higgs boson search at the LHC will be correlated, and their interplay will allow for a deep probe for SUSY. Focusing on the MSSM Higgs bosons, their observability at the LHC is studied [4] in the parameter space allowed by current dark matter and collider constraints.

Note that in this mini review we will not discuss the SUSY explanation for the PAMELA cosmic ray anomaly [8], which is subject to large uncertainty and could also be explained astrophysically by pulsars. Usually, the MSSM, NMSSM or nMSSM are hard to give a 
satisfactory explanation for such an anomaly (the final states of the neutralino dark matter annihilation will give excessive anti-protons or/and the large boost factor for the annihilation rate is hard to generate from the elegant Sommerfeld enhancement) [13, 14] while some general singlet extensions may give a plausible explanation [9 11] with some tension with the CDMS limits [12].

This review is organized as follows. In Sec III we recapitulate the three SUSY models: the MSSM, NMSSM and nMSSM. Then we discuss the current CDMS/XENON constraints on the parameter space of each model and their implication for the MSSM Higgs search at the LHC in Sec III. Finally, a summery is given in Sec. IV.

\section{SUSY MODELS}

The MSSM has the minimal content of particles and thus is the most economical realization of supersymmetry. So far this model has been most intensively studied. However, this model suffers from the so-called $\mu$-problem and the little hierarchy problem. Due to this reason, some singlet extensions like the NMSSM and nMSSM have recently attracted much effort. In both the NMSSM and the nMSSM the superpotential does not contain the $\mu$-term which is generated by the non-zero vev of the newly introduced singlet field. The little hierarchy can also be alleviated since the tree-level upper bound on the SM-like Higgs boson is pushed up and the stop is not necessarily so heavy to induce the large quantum effects for the Higgs mass. Meanwhile, the LEP II lower bound of $114 \mathrm{GeV}$ on the SM-like Higgs boson is also somewhat relaxed since this Higgs boson has the singlet mixture and its coupling with the $Z$-boson is weakened. The superpotentials of these models are given by

$$
\begin{aligned}
W_{\mathrm{MSSM}} & =W_{F}+\mu \hat{H}_{u} \cdot \hat{H}_{d}, \\
W_{\mathrm{NMSSM}} & =W_{F}+\lambda \hat{H}_{u} \cdot \hat{H}_{d} \hat{S}+\frac{1}{3} \kappa \hat{S}^{3}, \\
W_{\mathrm{nMSSM}} & =W_{F}+\lambda \hat{H}_{u} \cdot \hat{H}_{d} \hat{S}+\xi_{F} M_{n}^{2} \hat{S},
\end{aligned}
$$

where $W_{F}=Y_{u} \hat{Q} \cdot \hat{H}_{u} \hat{U}-Y_{d} \hat{Q} \cdot \hat{H}_{d} \hat{D}-Y_{e} \hat{L} \cdot \hat{H}_{d} \hat{E}$ with $\hat{Q}, \hat{U}$ and $\hat{D}$ being the squark superfields, and $\hat{L}$ and $\hat{E}$ being the slepton superfields, $\hat{H}_{u}$ and $\hat{H}_{d}$ are the Higgs doublet superfields, $\lambda, \kappa$ and $\xi_{F}$ are dimensionless coefficients, and $\mu$ and $M_{n}$ are parameters with mass dimension.

So in the MSSM we have the SM-like Higgs boson $h_{S M}$, the heavy neutral CP-even Higgs 
boson $H$ and CP-odd Higgs boson $A$ plus the charged Higgs bosons $H^{ \pm}$. In the NMSSM and nMSSM, we have one more CP-even Higgs boson and one more CP-odd Higgs boson. Because the lightest CP-even Higgs boson and the lightest CP-odd Higgs boson may be dominated by singlet components, they can escape the experimental constraints and thus can be very light (sometimes called the light dark Higgs).

The neutralinos are the mixture of neutral gauginos and neutral Higgsinos. While there are four neutralinos in the MSSM, the NMSSM and nMSSM each has five neutralinos. The lightest one is usually assumed to be the lightest superparticle (LSP). Due to the $R$-parity conservation, this LSP is stable. Since it has weak interaction and its mass is typically around the weak scale (a light LSP at GeV scale may be also possible, see, e.g, [15]), it is a perfect WIMP and serves as the dark matter particle. The neutralino mass matrices in these models are given by

$$
\left(\begin{array}{cccc}
M_{1} & 0 & m_{Z} s_{W} s_{b} & -m_{Z} s_{W} c_{b} \\
0 & M_{2} & -m_{Z} c_{W} s_{b} & m_{Z} c_{W} c_{b} \\
m_{Z} s_{W} s_{b} & -m_{Z} s_{W} s_{b} & 0 & -\mu \\
-m_{Z} s_{W} c_{b} & -m_{Z} c_{W} c_{b} & -\mu & 0
\end{array}\right)
$$

for the MSSM

$$
\left(\begin{array}{ccccc}
M_{1} & 0 & m_{Z} s_{W} s_{b} & -m_{Z} s_{W} c_{b} & 0 \\
0 & M_{2} & -m_{Z} c_{W} s_{b} & m_{Z} c_{W} c_{b} & 0 \\
m_{Z} s_{W} s_{b} & -m_{Z} s_{W} s_{b} & 0 & -\mu & -\lambda v c_{b} \\
-m_{Z} s_{W} c_{b} & -m_{Z} c_{W} c_{b} & -\mu & 0 & -\lambda v s_{b} \\
0 & 0 & -\lambda v c_{b} & -\lambda v s_{b} & 2 \frac{\kappa}{\lambda} \mu
\end{array}\right)
$$

for the NMSSM, and

$$
\left(\begin{array}{ccccc}
M_{1} & 0 & m_{Z} s_{W} s_{b} & -m_{Z} s_{W} c_{b} & 0 \\
0 & M_{2} & -m_{Z} c_{W} s_{b} & m_{Z} c_{W} c_{b} & 0 \\
m_{Z} s_{W} s_{b} & -m_{Z} s_{W} s_{b} & 0 & -\mu & -\lambda v c_{b} \\
-m_{Z} s_{W} c_{b} & -m_{Z} c_{W} c_{b} & -\mu & 0 & -\lambda v s_{b} \\
0 & 0 & -\lambda v c_{b} & -\lambda v s_{b} & 0
\end{array}\right)
$$

for the nMSSM. Here $M_{1}$ and $M_{2}$ are respectively $U(1)$ and $S U(2)$ gaugino masses, $s_{W}=$ $\sin \theta_{W}, c_{W}=\cos \theta_{W}, s_{b}=\sin \beta$ and $c_{b}=\cos \beta$ with $\tan \beta \equiv v_{u} / v_{d}$. 
Note the property of the neutralino LSP is determined by its components. In the MSSM with the GUT relation $M_{2} \simeq 2 M_{1}$, it may be bino-like or Higgsino-like (without the GUT relation it may also be wino-like). Since the LSP-nucleon scattering is dominated by exchanging the Higgs bosons (for heavy squarks), a bino-like neutralino scatters very weakly with the neucleon and thus difficult to detect at CDMS/XENON. In the NMSSM, in the most part of the parameter space the neutralino LSP is quite similar to the case of MSSM [3], but there exists some corner of the parameter space in which the neutralino LSP is singlino-like and thus can be very light [15]. In the nMSSM, due to the zero diagonal element for the singlino in the mass matrix Eq.(6) , the singlino is always very light and thus the neutralino LSP is singlino-like. Therefore, the property of the neutralino LSP in the nMSSM is quite different from the MSSM or NMSSM.

\section{NUMERICAL RESULTS AND DISCUSSIONS}

In our study we consider the following experimental constraints: (1) The direct bounds on sparticle and Higgs masses from LEP and Tevatron experiments; (2) The LEP II search for the Higgs bosons from various channels; (3) The LEP I and LEP II constraints on the productions of neutralinos and charginos; (4) The indirect constraints from the precision electroweak observables and various $B$-decay and mixings; (5) The constraint from the muon anomalous magnetic moment, for which we require the SUSY effects to account at $2 \sigma$ level; (6) The dark matter constraints from the WMAP relic density $(2 \sigma)$. For the CDMS II/XENON100 direct detection exclusion limits (90\% C.L.) on the scattering cross section, we will display the results with/without such limits. In addition to the above experimental limits, we also consider the constraint from the stability of the Higgs potential, which requires that the physical vacuum of the Higgs potential with non-vanishing vevs of Higgs scalars should be lower than any local minima.

In our scan the soft breaking parameters are assumed to be below $1 \mathrm{TeV}$, and the parameter $\lambda$ at weak scale is assumed to be less than about 0.7 to ensure the perturbativity of the theory up to the grand unification scale. Further, to reduce the number of the relevant soft parameters, we assumed the so-called $m_{h}^{\max }$ scenario with the soft masses for the third generation squarks: $M_{Q_{3}}=M_{U_{3}}=M_{D_{3}}=800 \mathrm{GeV}$, and $X_{t}=A_{t}-\mu \cot \beta=-1600 \mathrm{GeV}$.

The parameter space surviving the above constraints is plotted in Fig.1 of [3]. It shows 
that for each model the CDMS-II/XENON100 limits can exclude a large part of the parameter space allowed by other constraints and the future SuperCDMS (25 kg) or XENON100 (6000 kg-days) can cover most of the allowed parameter space. Still, some part of the allowed parameter space is beyond the future sensitivity of the SuperCDMS or XENON100. To totally cover the allowed parameter space of these SUSY models, a much larger detector or larger exposure is needed. From this figure we see that in the nMSSM the LSP can be as light as several GeV while for the MSSM and NMSSM the LSP mass has a lower bound of $50 \mathrm{GeV}$ which is from the assumed GUT relation $M_{1} \simeq 0.5 M_{2}$ (plus the chargino lower bound of $103.5 \mathrm{GeV}$ ). Without such a GUT relation, a lighter LSP is allowed. The similarity of the allowed parameter space between the MSSM and NMSSM is due to the fact that in the most part of the parameter space the LSP in both models is bino-like. The peculiarity of the nMSSM parameter space is due to the fact that the LSP is singlino-like in this model.

The property of the neutralino LSP in the allowed parameter space is shown in Fig.2 of [3]. It shows that in case of unobservation of dark matter the CDMS/XENON will push the neutralino LSP more bino-like for the MSSM and NMSSM while more singlino-like for the nMSSM. This can be understood easily because a more bino-like or singlino-like LSP scatters more feebly with the nucleon and more difficult to detect at the CDMS/XENON.

The charged Higgs boson mass range is shown Fig.7 of [3]. It shows that in case of unobservation of dark matter the CDMS/XENON will push up the charged Higgs boson mass for the MSSM and NMSSM. Then for the MSSM we studied the charged Higgs signal at the LHC $g g / g b \rightarrow t[b] H^{+}$with $H^{+}$subsequently decaying to $\tau^{+} \nu_{\tau}$ [4]. As shown in Fig.4 of [4], only a few survived points lie above the $5 \sigma$ discovery sensitivity obtained by the ATLAS collaboration for $30 \mathrm{fb}^{-1}$ integrated luminosity. Thus the likelihood for discovering the charged Higgs boson at the LHC is very small.

For the MSSM neutral Higgs bosons $H$ and $A$ at the LHC, we consider the channels $g g \rightarrow H(A)$ or $b \bar{b} H(A)$ with $H(A)$ decaying to $\tau$ leptons. The results are shown in Fig.5 of [4]. It shows that in the allowed parameter space with a large $\mu$ the neutral Higgs bosons $H$ and $A$ are quite likely observable at the LHC. This can be understood from the fact that for a larger $\mu$ parameter the neutralino LSP is more bino-like and scatters more feebly with the nucleon, which will weaken the CDMS/XENON constraints.

Finally, for the light Higgs bosons in the NMSSM, some very recent studies [16] showed that they may have some interesting signals at the LHC, e.g., the enhanced di-photon signal. 
Such enhanced di-photon signal may help to distinguish the NMSSM from the MSSM.

\section{CONCLUSION}

The current collider experiments and the dark matter relic density measurement stringently constrained the SUSY parameter space. The CDMS II and XENON100 limits further shrink the allowed parameter space and pushed the neutralino dark matter to be bino-like for the MSSM and NMSSM. The future sensitivity of the SuperCDMS or XENON100 can cover most of the allowed parameter space for each model and in case of un-observation of dark matter the neutralino dark matter will be more bino-like in the MSSM and NMSSM while more singlino-like in the nMSSM. In the currently allowed parameter space the MSSM charged Higgs boson is quite unlikely to be discovered at the LHC while the neutral Higgs bosons $H$ and $A$ may be accessible at the LHC in the parameter space with a large $\mu$ parameter.

Note added: When this manuscript is being prepared, more studies about light SUSY dark matter appeared in the arXiv [17].

\section{Acknowledgments}

This work was supported in part by the National Natural Science Foundation of China under grant Nos. 10821504, 10725526 and 10635030.

[1] Z. Ahmed et al., arXiv:0912.3592.

[2] E. Aprile et al., arXiv:1005.0380.

[3] J. Cao et al., JHEP 1007, 044 (2010).

[4] J. Cao et al., Phys. Rev. D 82, 051701 (2010).

[5] J. Ellis, K. A. Olive, Y. Santoso and V. C. Spanos, Phys. Rev. D 71, 095007 (2005).

[6] J. R. Ellis et al., Phys. Rev. D 39, 844 (1989); M. Drees, Int. J. Mod. Phys. A4, 3635 (1989); S. F. King and P. L. White, Phys. Rev. D 52, 4183 (1995); B. Ananthanarayan and P.N. Pandita, Phys. Lett. B 353, 70 (1995); B. A. Dobrescu and K. T. Matchev, JHEP 0009, 031 (2000); V. Barger, P. Langacker, H.-S. Lee and G. Shaughnessy, Phys. Rev. D 73, 115010 
(2006); R. Dermisek and J. F. Gunion, Phys. Rev. Lett. 95, 041801 (2005); G. Hiller, Phys. Rev. D 70, 034018 (2004); F. Domingo and U. Ellwanger, JHEP 0712, 090 (2007); Z. Heng et al., Phys. Rev. D 77, 095012 (2008); R. N. Hodgkinson and A. Pilaftsis, Phys. Rev. D 76, 015007 (2007); W. Wang, Z. Xiong and J. M. Yang, Phys. Lett. B 680, 167 (2009); J. Cao and J. M. Yang, JHEP 0812, 006 (2008); Phys. Rev. D 78, 115001 (2008).

[7] C. Panagiotakopoulos and K. Tamvakis, Phys. Lett. B 446, 224 (1999); C. Panagiotakopoulos and A. Pilaftsis, Phys. Rev. D 63, 055003 (2001); A. Dedes et al., Phys. Rev. D 63, 055009 (2001); J. Cao, H. E. Logan and J. M. Yang, Phys. Rev. D 79, 091701 (2009); J. Cao, Z. Heng and J. M. Yang, JHEP 1011, 110 (2010).

[8] O. Adriani et al., PAMELA Collaboration, Nature 458, 607 (2009).

[9] D. Hooper and T. M. P. Tait, Phys. Rev. D 80, 055028 (2009).

[10] W. Wang, Z. Xiong, J. M. Yang and L.-X. Yu, JHEP 0911, 053 (2009).

[11] J. M. Yang, Mod. Phys. Lett. A25 (2010) 976.

[12] C. Arina, F.-X. Josse-Michaux and N. Sahu, Phys. Lett. B 691 (2010) 219.

[13] P. Grajek et al., Phys. Rev. D 79 (2009) 043506.

[14] Y. Bai, M. Carena and J. Lykken, Phys. Rev. D 80 (2009) 055004.

[15] See, e.g., D. A. Vasquez et al., arXiv:1009.4380 [hep-ph]; P. Draper et al., arXiv:1009.3963 [hep-ph]; J. F. Gunion, A. V. Belikov and D. Hooper, arXiv:1009.2555 [hep-ph]; A. V. Belikov, J. F. Gunion, D. Hooper and T. Tait, arXiv:1009.0549 [hep-ph].

[16] U. Ellwanger, arXiv:1012.1201 [hep-ph]; M. Almarashi and S. Moretti, arXiv:1011.6547 [hep$\mathrm{ph}$.

[17] See, e.g., N. Fornengo, S. Scopel and A. Bottino, arXiv:1011.4743 [hep-ph]; Z. Kang et al., JCAP 1101, 028 (2011). 